\title{
A NOVEL DESIGN STRATEGY FOR STABLE METAL COMPLEXES OF NITROGEN MUSTARDS AS BIOREDUCTIVE PRODRUGS
}

Laurie L. Parker*, Stephen M. Lacy*, Louis J. Farrugia*, Cameron Evans*, David J. Robins* ${ }^{*}$, C. Caroline $\mathrm{O}^{\prime}$ Hare $^{\ddagger}$, John A. Hartley ${ }^{\ddagger}$, Mohammed Jaffar ${ }^{\S}$, Ian J. Stratford ${ }^{\S}$

* Department of Chemistry, University of Glasgow, Glasgow G12 8QQ, UK.

${ }^{\ddagger}$ Cancer Research UK, Drug-DNA Interactions Research Group, Department of Oncology, Royal Free and University College Medical School, UCL, 91 Riding House Street, London W1W 7BS, UK.

${ }^{\S}$ School of Pharmacy and Pharmaceutical Sciences, Coupland III Building, University of Manchester, Oxford Road, Manchester M13 9PL, UK.

\section{Contents:}

- RP-HPLC data for 3a and 3c

- Crystal data for 3a, 3c and 3d.

\section{HPLC spectra for 3a and 3c:}

3a-blank; 214, 280, $618 \mathrm{~nm}$

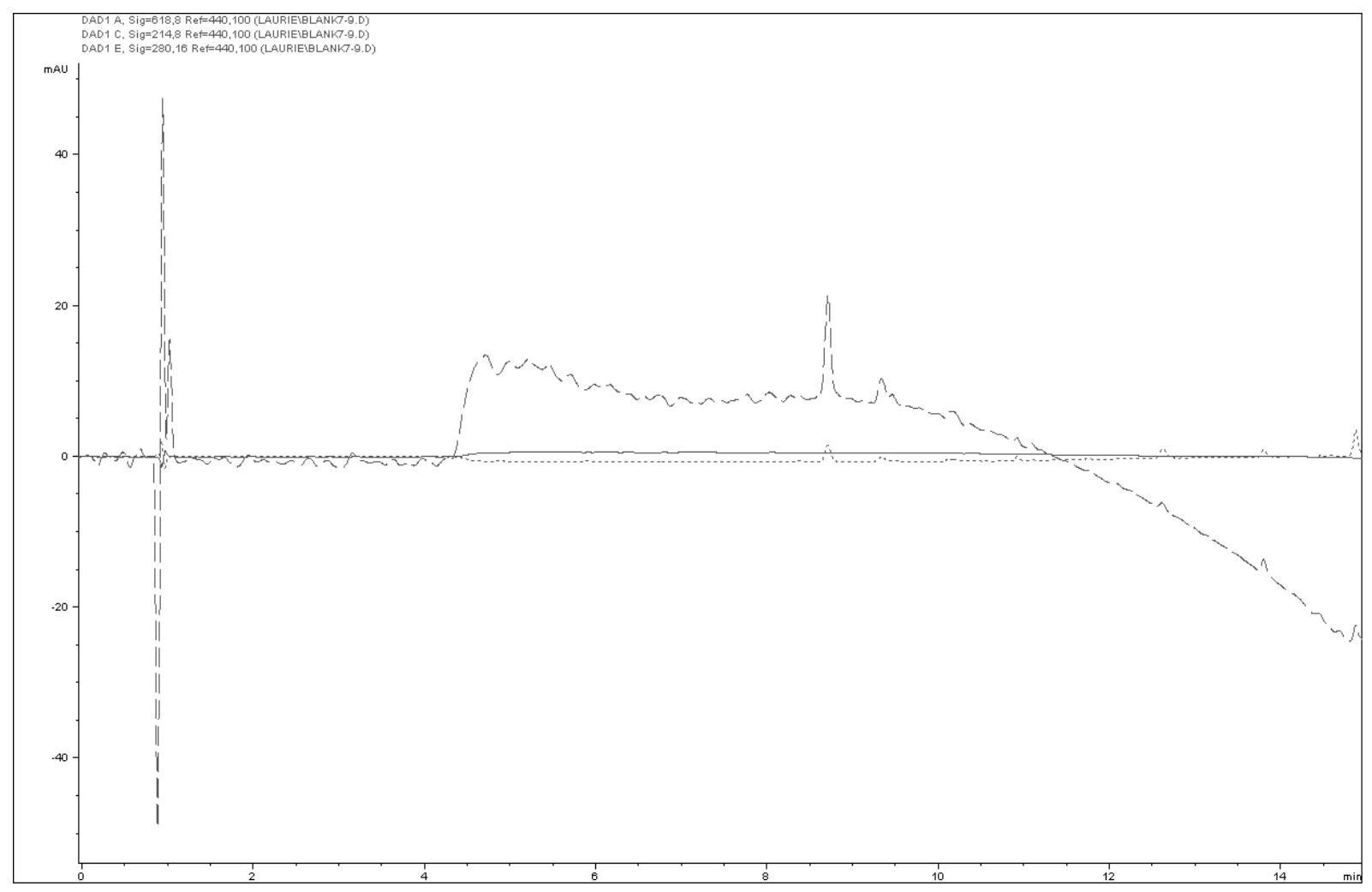

${ }^{1}$ Corresponding author. Tel. +44-141-330-4378; fax +44-141-330-4888; email: d.robins@chem.gla.ac.uk 
3a-sample; 214, $280,618 \mathrm{~nm}$

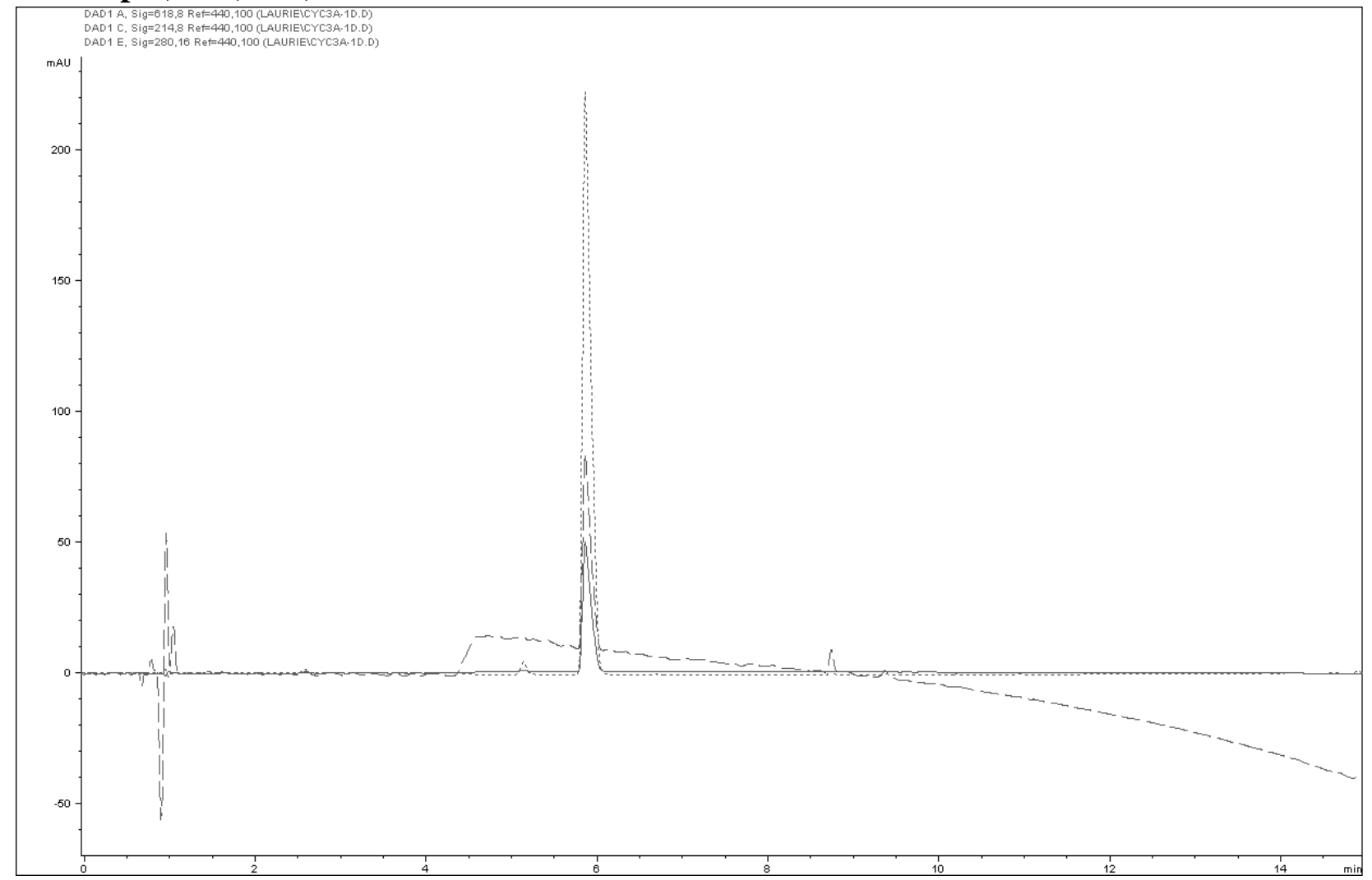

$3 a-280 \mathrm{~nm}$ : integrated non-blank peaks for \% purity

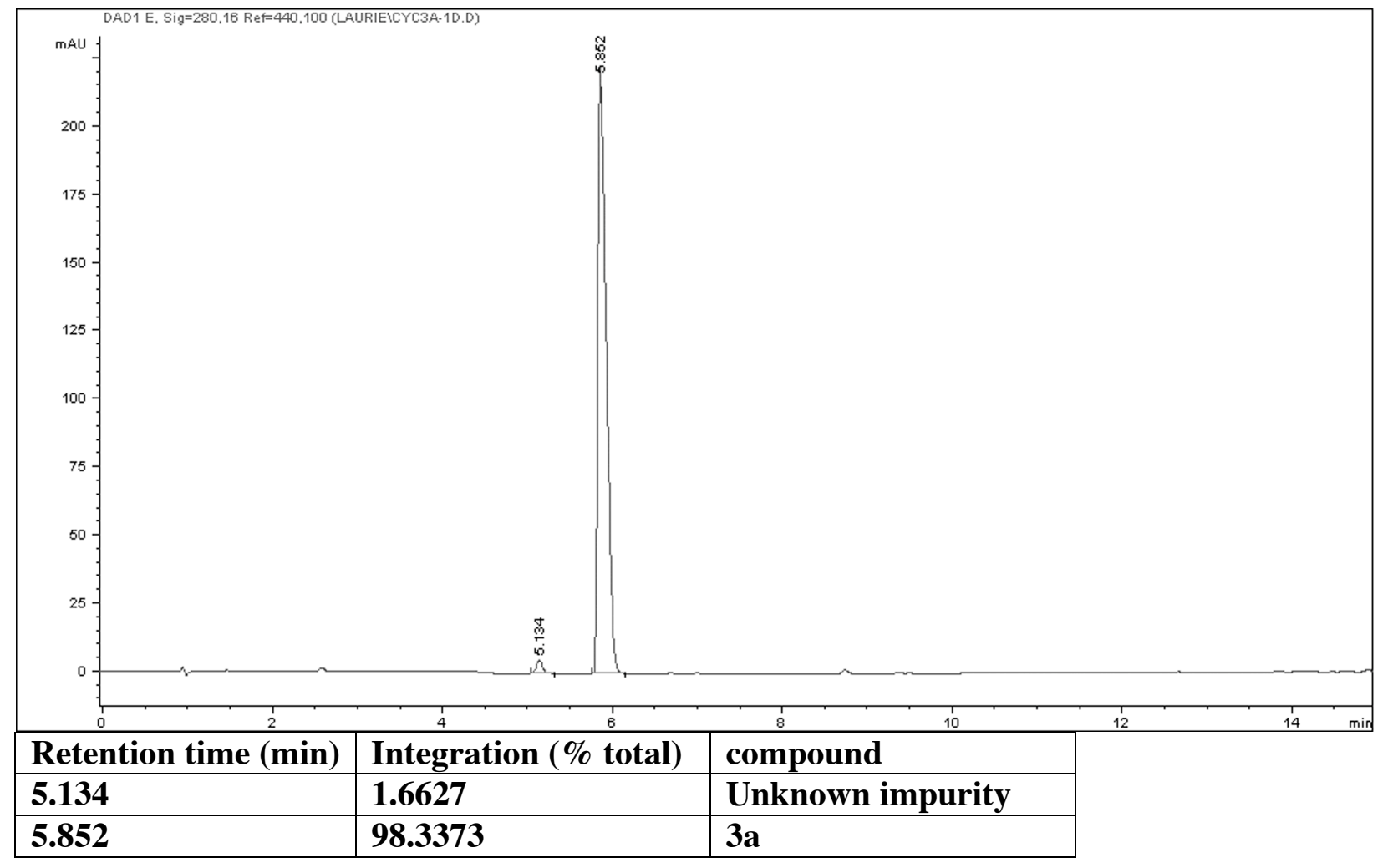




\section{3c-blank (280 nm)}

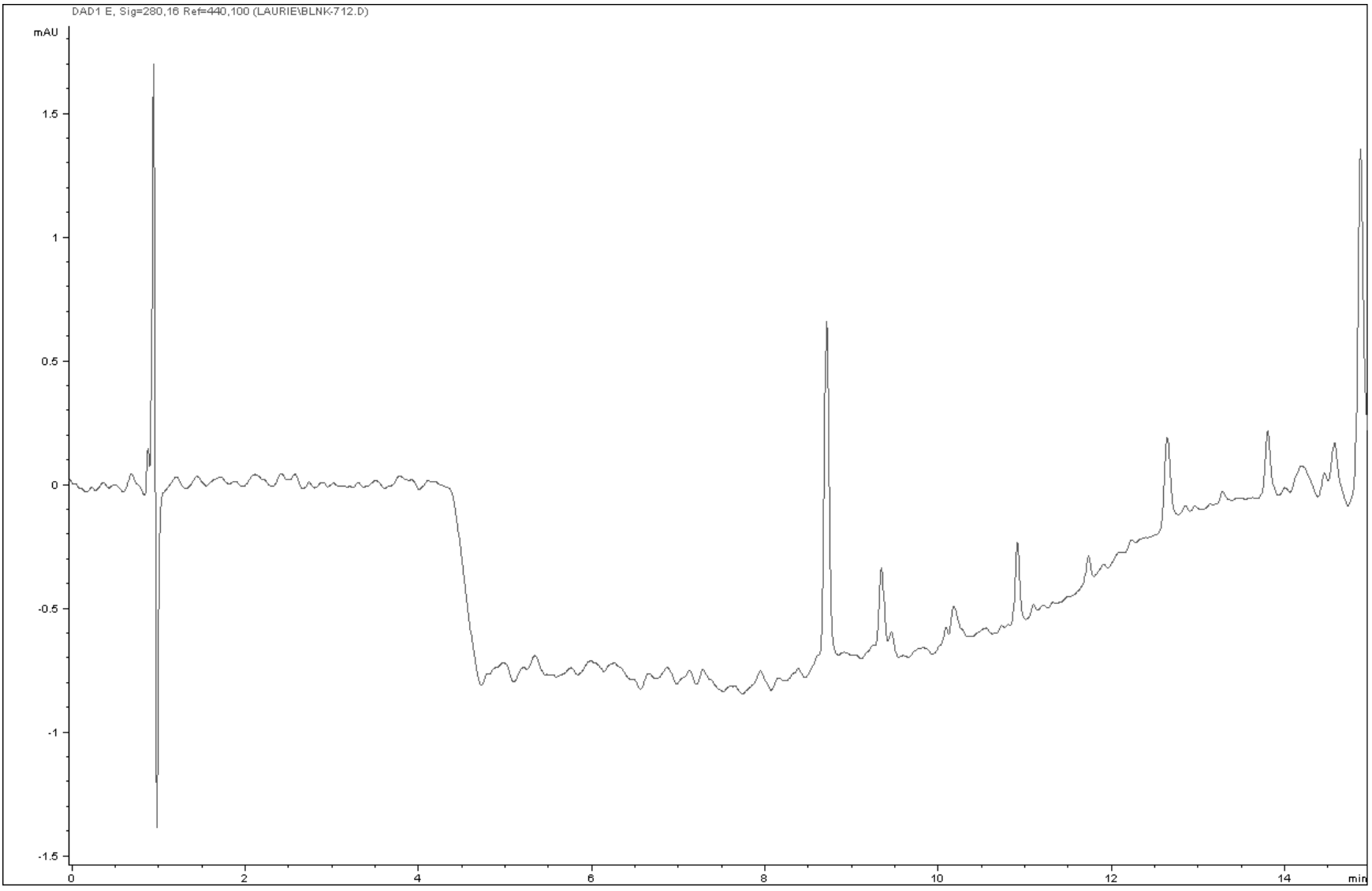

3c-sample; 214, 280, 590, $630 \mathrm{~nm}$

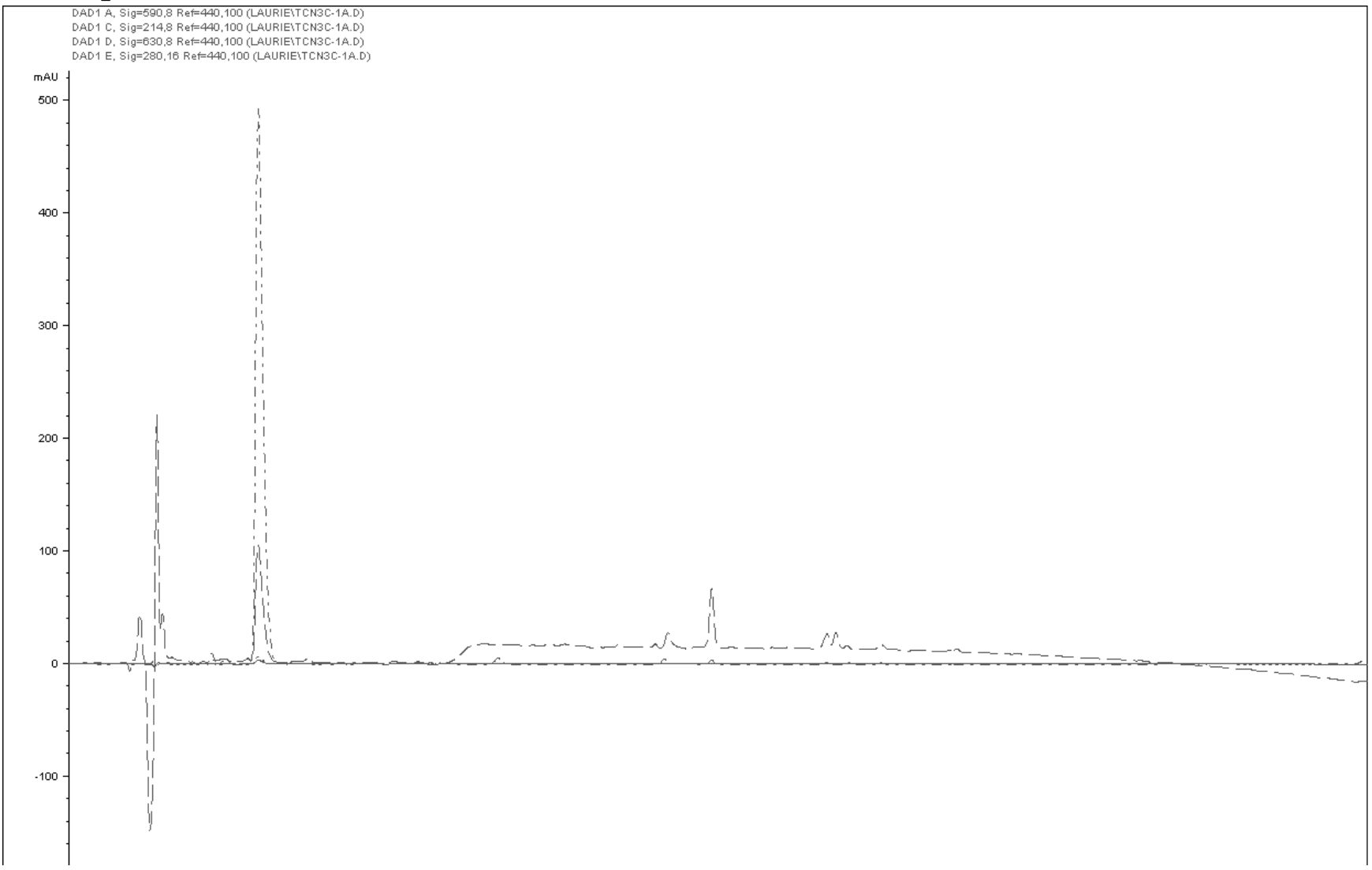


3a-280 nm: integrated non-blank peaks for \% purity

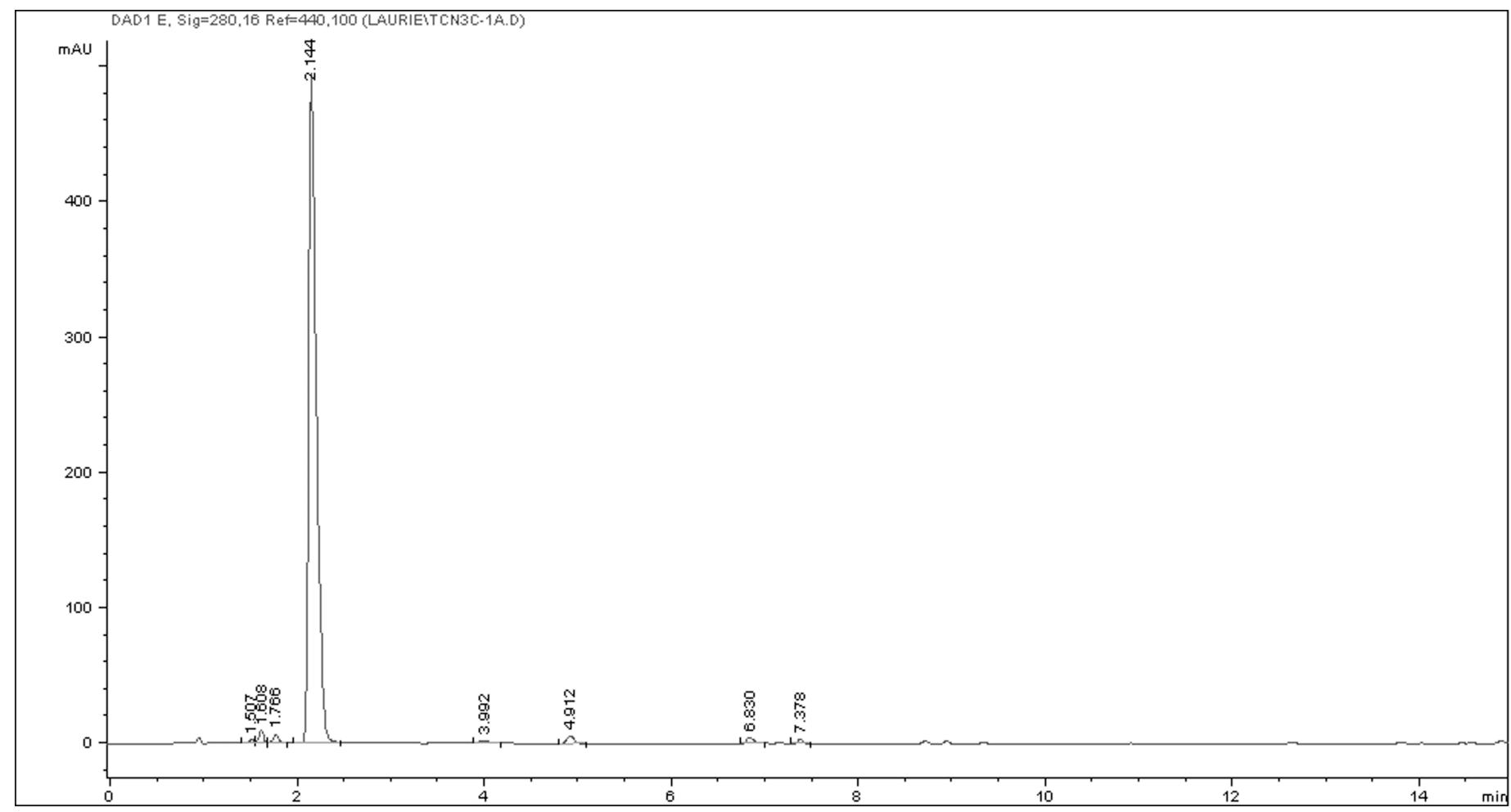

\begin{tabular}{|l|l|l|}
\hline Retention time (min) & Integration (\% total) & compound \\
\hline 1.507 & 0.4056 & Unknown impurity \\
\hline 1.608 & 1.0711 & Unknown impurity \\
\hline 1.766 & 0.8399 & Unknown impurity \\
\hline 2.144 & $\mathbf{9 4 . 5 0 7 1}$ & 3c \\
\hline 3.992 & 0.4795 & Unknown impurity \\
\hline 4.912 & 1.1697 & Unknown impurity \\
\hline 6.830 & 1.0142 & Unknown impurity \\
\hline 7.378 & 0.5129 & Unknown impurity \\
\hline
\end{tabular}




\section{CIF data for 3a:}

\# CIF produced by WinGX routine CIF_UPDATE

\# Created on 2002-09-17 at 12:20:45

\# Using CIFtbx version 2.6.2 16 Jun 1998

\# Dictionary name : cif core.dic

\# Dictionary vers : 2.2

\# Request file : c:|wingx \files $\backslash$ archive.dat

\# CIF files read : ljf089 dreduc gaussian sortav struct

data_ljf089

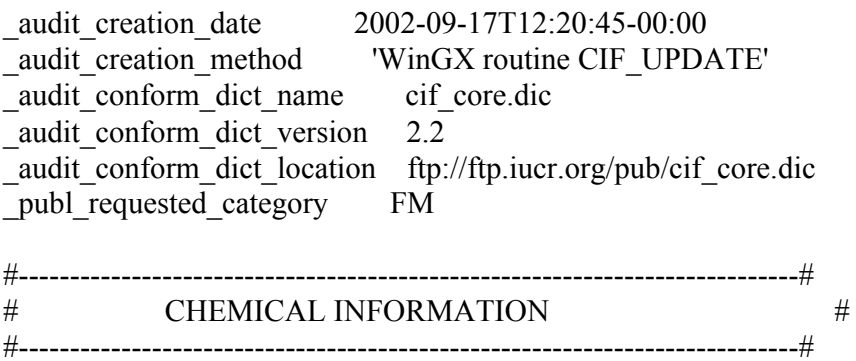

_chemical_name_systematic

?

_chemical_formula_moiety

_chemical_formula_sum

chemical_formula_weight

_chemical_compound_source

'C16 H32 Cl5 Cu N4, B F4'

'C16 H32 B Cl5 Cu F4 N4'

608.06

'synthesis as described'

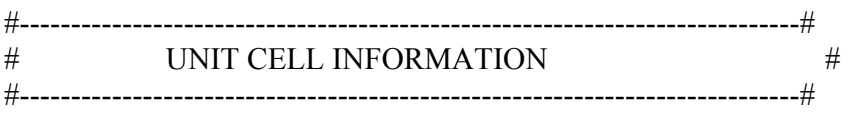

_symmetry_cell_setting

_symmetry_space_group_name_H-M

monoclinic

_symmetry_space_group_name_Hall

_symmetry_Int_Tables_number

'P 21/c'

'-P 2ybc'

loop_

symmetry_equiv_pos_as_xyz

'x, y, z'

'-x, $y+1 / 2,-z+1 / 2$ '

'-x, $-\mathrm{y},-\mathrm{z}$

'x, $-\mathrm{y}-1 / 2, \mathrm{z}-1 / 2$ '

cell_length_a

$15.0036(2)$

_cell_length_b

$8.33250(10)$

cell_length_c

cell_angle_alpha

20.3516(3)

_cell_angle_beta

cell_angle_gamma

90

cell volume

104.1620(10)

90

cell_formula_units_Z

$2466.98(6)$

_cell_measurement_temperature

ccell_measurement_reflns_used

_cell_measurement_theta_min

_cell_measurement_theta_max

_cell_measurement_wavelength

$150(2)$

5618

1

27.485

0.71073

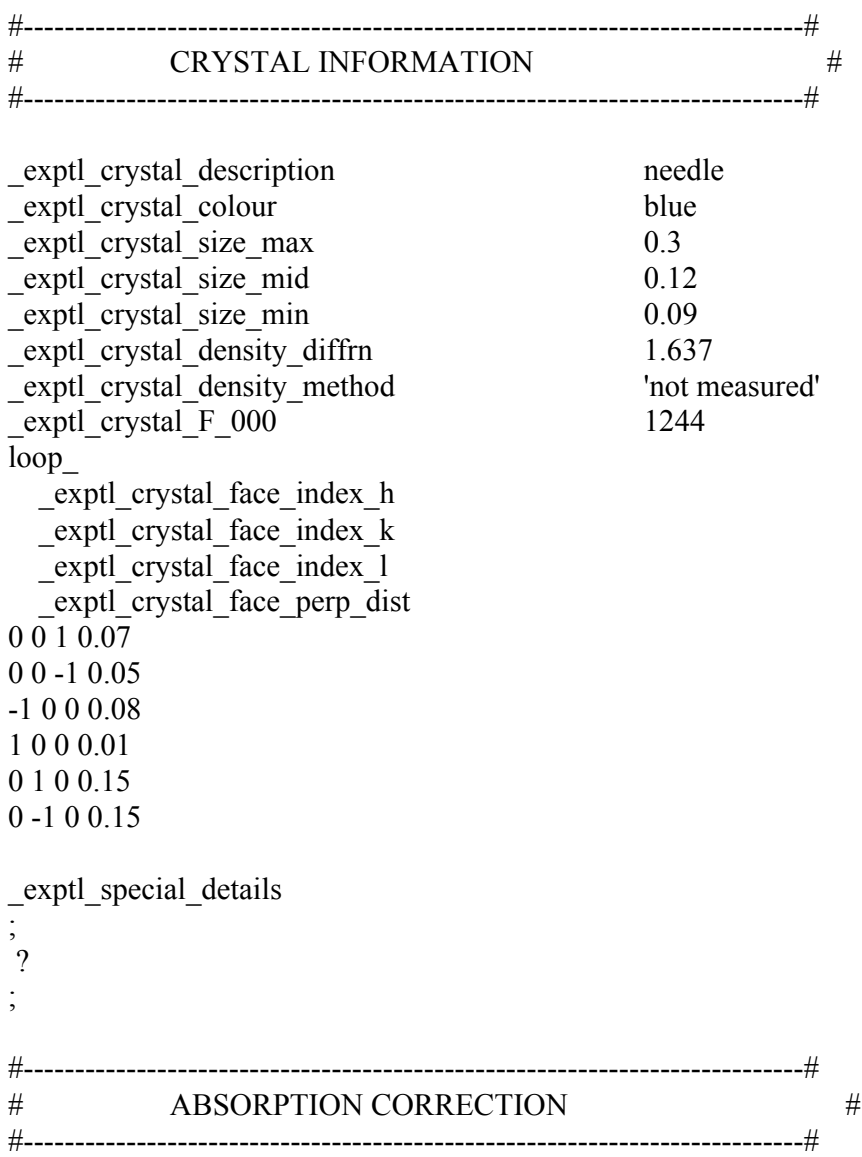

_exptl_absorpt_coefficient_mu $\quad 1.47$

_exptl_absorpt_correction_type gaussian exptl_absorpt_process_details ;

P. Coppens, L. Leiserowitz, D Rabinovich, Acta Cryst. (1965), 18, 1035-1038

;

exptl_absorpt_correction_T_min $\quad 0.672$

_exptl_absorpt_correction_T_max 0.882

\# \#- DATA COLLECTION

diffrn_source

'Enraf Nonius FR590'

_diffrn_ambient_temperature

$150(2)$

diffrn_radiation_wavelength

0.71073

_diffrn_radiation_type

diffrn_radiation_monochromator

_diffrn_radiation_probe

diffrn_detector

_diffrn_orient_matrix_type

scalepack cell' diffrn orient matrix ub 11

diffrn_orient_matrix_ub_12

diffrn_orient_matrix_ub_13

diffrn orient matrix ub 21

_diffrn_orient_matrix_ub_22
MoKla

graphite

$\mathrm{x}$-ray

'CCD plate'

'by Nonius Collect from

$0.515955 \mathrm{E}-1$

$-0.645854 \mathrm{E}-1$

$-0.95328 \mathrm{E}-2$

$0.395019 \mathrm{E}-1$

$0.981773 \mathrm{E}-1$ 
diffrn orient matrix ub 23

_diffrn_orient_matrix_ub_31

_diffrn_orient_matrix_ub_32

diffrn_orient_matrix_ub_33

_diffrn_measurement_device

goniostat'

diffrn measurement_device type

_diffrn_measurement_method

slices'

diffrn_reflns_av_R_equivalents

_diffrn_reflns_av_unetI/netI

_diffrn_reflns_number

diffrn_reflns limit_h min

_diffrn_reflns_limit_h_max

_diffrn_reflns_limit_k_min

diffrn reflns limit $\mathrm{k}$ max

_diffrn_reflns_limit_1_min

_diffrn_reflns_limit_1_max

_diffrn_reflns theta_min

_diffrn_reflns_theta_max

_diffrn_reflns_theta_full

27.52

27.52

0.995

0.995

5651

4620

$>2$ sigma(I)

\section{$-0.243479 \mathrm{E}-1$ \\ $95 \mathrm{~mm}$ CCD camera on $\backslash \mathrm{k}$ -}

Reflection file from gaussian, empirical correction in SORTAV used to remove anisotropy from mounting medium.

Scaled and merged with SORTAV

R.H. Blessing, (1987) Cryst. Rev. 1, 3-58

R.H. Blessing, (1989) J. Appl. Cryst. 22, 396-397

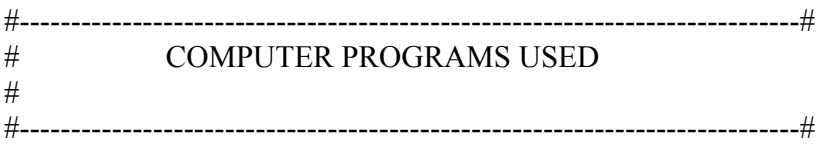

computing data collection

'Collect (Nonius BV, 1997-2000)'

computing_cell_refinement

'HKL Scalepack (Otwinowski \& Minor 1997)'

computing_data_reduction

'HKL Denzo and Scalepack (Otwinowski \& Minor 1997)'

computing structure_solution

'DIRDIF-99 (Beurskens et al, 1999)'

computing_structure_refinement

'SHELXL-97 (Sheldrick, 1997)'

computing_molecular_graphics

'Ortep-3 for Windows (Farrugia, 1997)'

computing publication material

'WinGX publication routines (Farrugia, 1999)' _reflns_threshold_expression

_diffrn_reflns_reduction_process

;

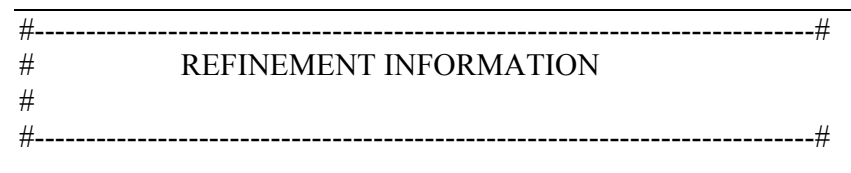

_refine_special_details

Refinement of $\mathrm{F}^{\wedge} 2^{\wedge}$ against ALL reflections. The weighted R-factor $w R$ and goodness of fit $\mathrm{S}$ are based on $\mathrm{F}^{\wedge} 2^{\wedge}$, conventional R-factors $\mathrm{R}$ are based on $\mathrm{F}$, with $\mathrm{F}$ set to zero for negative $\mathrm{F}^{\wedge} 2^{\wedge}$. The threshold expression of $\mathrm{F}^{\wedge} 2^{\wedge}>2 \operatorname{sigma}\left(\mathrm{F}^{\wedge} 2^{\wedge}\right)$ is used only for calculating $\mathrm{R}$ factors(gt) etc. and is not relevant to the choice of reflections for refinement. R-factors based on $\mathrm{F}^{\wedge} 2^{\wedge}$ are statistically about twice as large as those based on F, and R- factors based on ALL data will be even larger.

Both the cation and anion are disordered. The BF4 anion is disordered over two sites, the population was initially refined, but in the final cycles fixed at 50:50. The geometry of each component was restrained to tertrahedral with a common B-F distance refined.

The cation is partially disordered around the macrocyclic ring. The positions of the "arm" atoms either coincide or are too close to be resolved. The disorder population was refined. The $\mathrm{C}$ atoms of the minor component were refined with a common isotropic thermal parameter.

_refine_ls_structure_factor_coef

Fsqd $\begin{array}{lr}\text { refine_ls_matrix_type } & \text { full } \\ \text { refine_ls_weighting_scheme } & \text { calc } \\ \text { _refine_ls_weighting_detail } & \text { 'calc }\end{array}$

\begin{tabular}{|c|c|}
\hline atom sites solut & direct \\
\hline _atom_sites_solution_secondary & difmap \\
\hline _atom_sites_solution_hydrogens & geom \\
\hline refine_ls_hydrogen_treatment & mixed \\
\hline refine_ls_extinction_method & none \\
\hline refine_ls_number_reflns & 5651 \\
\hline refine_ls_number_parameters & 364 \\
\hline refine_1s_number_restraints & 20 \\
\hline refine_ls_R_factor_all & 0.0435 \\
\hline refine_ls_R_factor_gt & 0.0296 \\
\hline refine_ls_w $\overline{\mathrm{R}}$ _factor_ref & 0.068 \\
\hline refine_ls_wR_factor_gt & 0.0637 \\
\hline refine_1s_goodness_of_fit_ref & 1.039 \\
\hline refine_ls_restrained_S_all & 1.042 \\
\hline refine_ls_shift/su_max & 0.001 \\
\hline refine_ls_shift/su_mean & 0 \\
\hline refine_diff_density_max & 0.418 \\
\hline refine_diff_density_min & -0.341 \\
\hline refine_diff_density_rms & 0.056 \\
\hline
\end{tabular}

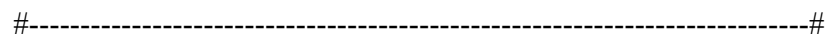

\# ATOMIC TYPES, COORDINATES AND THERMAL PARAMETERS \#

full
calc
'calc
$\mathrm{W}^{\prime} 1 /\left[\backslash \mathrm{s}^{\wedge} 2^{\wedge}\left(\mathrm{Fo}^{\wedge} 2^{\wedge}\right)+(0.0262 \mathrm{P})^{\wedge}\right.$
$\left.2^{\wedge}+1.6807 \mathrm{P}\right]$ where
$\mathrm{P}=\left(\mathrm{Fo}^{\wedge} 2^{\wedge}+2 \mathrm{Fc}^{\wedge} 2^{\wedge}\right) / 3^{\prime}$
direct
difmap
geom
mixed
none
5651
364
20
0.0435
0.0296
0.068
0.0637
1.039
1.042
0.001
0
0.418
-0.341
0.056


loop

_atom_type_symbol

_atom_type_description

atom type scat dispersion real

_atom_type_scat_dispersion_imag

_atom_type_scat_source

$\mathrm{Cu}^{-} \mathrm{Cu} 0 . \overline{3} 201 \overline{1} .2651$ 'International Tables Vol C Tables 4.2.6.8 and 6.1.1.4' $\mathrm{Cl} \mathrm{Cl} \mathrm{0.1484} \mathrm{0.1585} \mathrm{'International} \mathrm{Tables} \mathrm{Vol} \mathrm{C} \mathrm{Tables} \mathrm{4.2.6.8} \mathrm{and} \mathrm{6.1.1.4'}$ B B 0.0013 0.0007 'International Tables Vol C Tables 4.2.6.8 and 6.1.1.4' F F 0.0171 0.0103 'International Tables Vol C Tables 4.2.6.8 and 6.1.1.4' N N 0.0061 0.0033 'International Tables Vol C Tables 4.2.6.8 and 6.1.1.4' C C 0.0033 0.0016 'International Tables Vol C Tables 4.2.6.8 and 6.1.1.4' H H 00 'International Tables Vol C Tables 4.2.6.8 and 6.1.1.4'

loop

atom site label

_atom_site_type_symbol

_atom_site_fract_x

-atom_site_fract_y

_atom_site_fract_z

_atom_site_U_iso_or_equiv

_atom_site_adp_type

_atom_site_occupancy

_atom_site_symmetry_multiplicity

_atom_site_calc_flag

_atom_site_refinement_flags

_atom_site_disorder_assembly

_atom_site_disorder_group

$\mathrm{Cu} \overline{1} \mathrm{Cu} 0.213843(14)-0.40058(3)-0.178721(11) 0.02078$ (7) Uani $11 \mathrm{~d} . .$. Cl1 Cl -0.09069(3) -0.60654(7) -0.08402(3) 0.03610(12) Uani $11 \mathrm{~d} \ldots$ $\mathrm{Cl} 2 \mathrm{Cl} 0.23570(4) 0.03853(7) 0.03122(3) 0.04694(15)$ Uani $11 \mathrm{~d} \ldots$ $\mathrm{Cl} 3 \mathrm{Cl} 0.33468(5)$ 0.03514(7) $-0.35075(3)$ 0.04868(15) Uani $11 \mathrm{~d} \ldots$ $\mathrm{Cl} 4 \mathrm{Cl}$ 0.00589(4) $-0.61688(6)-0.43278(2)$ 0.03299(12) Uani $11 \mathrm{~d} \ldots$ Cl5 Cl 0.09823(3) -0.20626(6) -0.21712(2) 0.02612(10) Uani $11 \mathrm{~d}$. E . C1A C 0.06639(12) -0.5358(2) -0.11959(9) 0.0241(4) Uani $11 \mathrm{~d}$. . H1A1 H $0.0331-0.5408-0.1680 .029$ Uiso 11 calc R A 1 H1A2 H $0.0562-0.4278-0.10250 .029$ Uiso 11 calc R A 1 C1B C 0.02590(14) -0.6608(3) -0.08047(12) 0.0355(5) Uani $11 \mathrm{~d}$. E . H1B1 H $0.062-0.6646-0.03280 .043$ Uiso 11 calc R . . H1B2 H $0.028-0.7683-0.10080 .043$ Uiso 11 calc R . . C4A C 0.25210(13) -0.1747(2) -0.06421(10) 0.0270(4) Uani $11 \mathrm{~d} \ldots$ H4A1 H $0.1905-0.2102-0.06070 .032$ Uiso 11 calc R B 1 H4A2 H $0.2433-0.0853-0.09720 .032$ Uiso 11 calc R B 1 C4B C 0.30466(15) -0.1120(3) 0.00463(11) 0.0345(5) Uani $11 \mathrm{~d}$. E . H4B1 H $0.3641-0.06550 .00130 .041$ Uiso 11 calc R . . H4B2 H $0.3172-0.20080 .0380 .041$ Uiso 11 calc R . . C7A C 0.30124(13) -0.1723(2) -0.25699(10) 0.0291(4) Uani $11 \mathrm{~d} \ldots$ H7A1 H $0.2943-0.0936-0.22220 .035$ Uiso 11 calc R C 1 H7A2 H $0.243-0.1733-0.29260 .035$ Uiso 11 calc R C 1 C7B C 0.37795(16) -0.1155(3) -0.28835(12) 0.0408(5) Uani $11 \mathrm{~d}$. E . H7B1 H $0.402-0.2073-0.30970 .049$ Uiso 11 calc R . . H7B2 H $0.429-0.0709-0.25270 .049$ Uiso 11 calc R . C10A C 0.11457(13) -0.5414(2) -0.31101(9) 0.0243(4) Uani $11 \mathrm{~d} \ldots$ H10A H $0.1374-0.4489-0.33270 .029$ Uiso 11 calc R D 1 H10B H $0.0572-0.5078-0.29910 .029$ Uiso 11 calc R D 1 C10B C 0.09337(14) -0.6796(2) -0.36131(10) 0.0297(4) Uani $11 \mathrm{~d}$. E . H10C H $0.0722-0.7741-0.33980 .036$ Uiso 11 calc R. . H10D H $0.1494-0.7101-0.37580 .036$ Uiso 11 calc R . . N1 N 0.16589(18) -0.5571(3) -0.11417(12) 0.0248(6) Uani 0.780(3) 1 d P E 1

N4 N 0.2971(2) -0.3092(4) -0.09089(17) 0.0261(7) Uani 0.780(3) 1 d P E 1
N7 N 0.31538(19) -0.3329(4) -0.22545(12) 0.0293(7) Uani 0.780(3) 1 d P E 1 $\mathrm{N} 10 \mathrm{~N} 0.18358(16)-0.5846(3)-0.24860(12) 0.0246(5)$ Uani 0.780(3) $1 \mathrm{~d}$ P E 1 C2 C 0.22319(19) -0.5284(4) -0.04352(13) 0.0294(6) Uani 0.780(3) $1 \mathrm{~d}$ P E 1 H2A H $0.2341-0.6314-0.01850 .035$ Uiso $0.780(3) 1$ calc PR E 1 H2B H $0.1901-0.4558-0.01910 .035$ Uiso $0.780(3) 1$ calc PR E 1 C3 C 0.31382(19) -0.4545(3) -0.04638(13) 0.0301(6) Uani 0.780(3) 1 d P E 1 H3A H $0.3485-0.4239-0.00020 .036$ Uiso $0.780(3) 1$ calc PR E 1 H3B H $0.3509-0.5334-0.06450 .036$ Uiso $0.780(3) 1$ calc PR E 1 C5 C 0.38512(17) -0.2566(4) -0.10680(15) 0.0323(6) Uani 0.780(3) 1 d P E 1 H5A H $0.4372-0.2785-0.06740 .039$ Uiso $0.780(3) 1$ calc PR E 1 H5B H $0.383-0.1395-0.11540 .039$ Uiso $0.780(3) 1$ calc PR E 1 C6 C 0.39999(17) -0.3446(4) -0.16856(14) 0.0348(7) Uani 0.780(3) 1 d P E 1 H6A H $0.4528-0.297-0.18270 .042$ Uiso $0.780(3) 1$ calc PR E 1 H6B H $0.4142-0.4588-0.15710 .042$ Uiso $0.780(3) 1$ calc PR E 1 C8 C 0.3170(2) -0.4598(4) -0.27809(14) 0.0342(7) Uani 0.780(3) 1 d P E 1 H8A H $0.2818-0.4217-0.3230 .041$ Uiso $0.780(3) 1$ calc PR E 1 H8B H $0.3812-0.479-0.28060 .041$ Uiso $0.780(3) 1$ calc PR E 1 C9 C 0.27595(18) -0.6138(4) -0.26080(15) 0.0337(7) Uani 0.780(3) 1 d P E 1 H9A H $0.2706-0.6911-0.29850 .04$ Uiso 0.780(3) 1 calc PR E 1 H9B H $0.3168-0.6613-0.21970 .04$ Uiso $0.780(3) 1$ calc PR E 1 C11 C 0.1519(2) -0.7270(3) -0.21484(15) 0.0303(6) Uani 0.780(3) 1 d P E 1 H11A H $0.174-0.8271-0.23180 .036$ Uiso $0.780(3) 1$ calc PR E 1 H11B H $0.0839-0.7299-0.22610 .036$ Uiso $0.780(3) 1$ calc PR E 1 C12 C 0.1886(2) -0.7164(3) -0.13898(14) 0.0314(6) Uani 0.780(3) 1 d P E 1 H12A H $0.1611-0.8025-0.11680 .038$ Uiso 0.780 (3) 1 calc PR E 1 H12B H $0.2561-0.7315-0.12720 .038$ Uiso 0.780 (3) 1 calc PR E 1 N1A N 0.1588(7) $-0.5847(13)-0.1347(5)$ 0.0180(8) Uiso 0.220(3) 1 d P E 2 N4A N 0.2844(10) -0.3370(17) -0.0820(7) 0.0180(8) Uiso 0.220(3) $1 \mathrm{~d}$ P E 2 N7A N 0.3319(8) -0.3114(13) -0.2073(5) 0.0180(8) Uiso 0.220(3) $1 \mathrm{~d}$ P E 2 N10A N 0.2098(6) $-0.5578(11)-0.2584(5) 0.0180(8)$ Uiso 0.220(3) $1 \mathrm{~d}$ P E 2 C2A C 0.2305(5) -0.6093(10) -0.0714(4) 0.0180(8) Uiso 0.220(3) $1 \mathrm{~d}$ P E 2 H2A 1 H $0.2847-0.6611-0.08210 .022$ Uiso $0.220(3) 1$ calc PR E 2 H2A2 H $0.2068-0.6822-0.04120 .022$ Uiso $0.220(3) 1$ calc PR E 2 C3A C 0.2593(7) -0.4532(11) -0.0353(4) 0.0180(8) Uiso 0.220(3) $1 \mathrm{~d}$ P E 2 H3A1 H $0.2082-0.4098-0.01780 .022$ Uiso 0.220(3) 1 calc PR E 2 H3A2 H $0.3125-0.47160 .00370 .022$ Uiso 0.220(3) 1 calc PR E 2 C5A C 0.3805(5) -0.3420(11) -0.0826(4) 0.0180(8) Uiso 0.220(3) $1 \mathrm{~d}$ P E 2 H5A1 H $0.4003-0.455-0.08390 .022$ Uiso 0.220(3) 1 calc PR E 2 H5A2 H $0.4178-0.2923-0.04060 .022$ Uiso 0.220(3) 1 calc PR E 2 C6A C 0.3965(6) -0.2525(11) -0.1441(5) 0.0180(8) Uiso 0.220(3) 1 d P E 2 H6A1 H $0.387-0.1361-0.13890 .022$ Uiso 0.220(3) 1 calc PR E 2 H6A2 H $0.4606-0.2691-0.14730 .022$ Uiso 0.220(3) 1 calc PR E 2 C8A C 0.3670(5) -0.4525(9) -0.2352(4) 0.0180(8) Uiso 0.220(3) 1 d P E 2 H8A1 H $0.3938-0.5286-0.19830 .022$ Uiso 0.220(3) 1 calc PR E 2 H8A2 H $0.4161-0.4201-0.25740 .022$ Uiso $0.220(3) 1$ calc PR E 2 C9A C 0.2887(6) -0.5341(13) -0.2870(5) 0.0180(8) Uiso 0.220(3) $1 \mathrm{~d}$ P E 2 H9A1 H $0.2707-0.4668-0.32820 .022$ Uiso 0.220(3) 1 calc PR E 2 H9A2 H $0.3099-0.6391-0.30020 .022$ Uiso $0.220(3) 1$ calc PR E 2 C11A C 0.2171(6) -0.7167(9) -0.2238(4) 0.0180(8) Uiso 0.220(3) $1 \mathrm{~d}$ P E 2 H11C H $0.2799-0.7308-0.19470 .022$ Uiso 0.220(3) 1 calc PR E 2 H11D H $0.2054-0.8036-0.25790 .022$ Uiso $0.220(3) 1$ calc PR E 2 C12A C 0.1475(6) -0.7265(10) -0.1807(6) 0.0180(8) Uiso 0.220(3) $1 \mathrm{~d}$ P E 2 H12C H $0.0844-0.7284-0.21030 .022$ Uiso 0.220 (3) 1 calc PR E 2 H12D H $0.1572-0.8265-0.15360 .022$ Uiso 0.220(3) 1 calc PR E 2 B1 B 0.4673(3) 0.1679(6) 0.8726(2) 0.0370(18) Uani 0.5 1 d PD F 3 F11 F 0.5209(3) 0.0302(5) 0.8827(3) 0.0434(13) Uani 0.51 d PD F 3 F12 F 0.5144(3) 0.2927(5) 0.9107(2) 0.0453(11) Uani 0.5 1 d PD F 3 F13 F 0.3862(2) 0.1380(4) 0.89231(18) 0.0510(9) Uani 0.51 d PD F 3 F14 F 0.44669(18) 0.2078(4) 0.80444(12) 0.0544(7) Uani 0.51 d PD F 3 B2 B 0.4644(3) 0.1548(6) 0.8915(2) 0.0385(18) Uani 0.5 1 d PD G 4 F21 F 0.4682(2) 0.0915(5) 0.95508(15) 0.0804(11) Uani $0.51 \mathrm{~d}$ PD G 4 F22 F 0.3770(2) 0.1290(5) 0.85117(19) 0.0636(11) Uani $0.51 \mathrm{~d}$ PD G 4 
F23 F 0.5285(3) 0.0727(6) 0.8664(3) 0.0473(13) Uani 0.5 1 d PD G 4 F24 F 0.4849(3) 0.3161(5) 0.8974(3) 0.086(2) Uani 0.5 1 d PD G 4

loop_ _atom_site_aniso_label _atom_site_aniso_U_11 -atom_site_aniso_U_22 _atom_site_aniso_U_33 _atom_site_aniso_U_23 -atom_site_aniso_U_13 atom_site_aniso_U_12

$\mathrm{Cu} 10.02042(\overline{12}) 0 . \overline{02506(12)} 0.01689(12)-0.00053(9) 0.00463(9)$ $0.00141(9)$

C11 0.0325(3) 0.0440(3) 0.0352(3) 0.0030(2) 0.0148(2) $-0.0040(2)$ $\mathrm{Cl} 20.0638(4) 0.0455(3) 0.0312(3)-0.0071(2) 0.0109(3) 0.0156(3)$ $\mathrm{Cl} 30.0660$ (4) 0.0431(3) 0.0468(3) 0.0072(3) 0.0327(3) 0.0022(3) Cl4 0.0401(3) 0.0377(3) 0.0193(2) $-0.00126(19) 0.0035(2)-0.0060(2)$ Cl5 0.0249(2) 0.0298(2) 0.0237(2) 0.00260(18) 0.00597(18) 0.00548(18) C1A 0.0253(9) 0.0254(9) 0.0230(9) 0.0014(8) 0.0085(8) 0.0015(8) C1B 0.0316(11) 0.0333(11) 0.0431(13) 0.0101(10) 0.0122(10) 0.0012(9) C4A 0.0267(10) 0.0310(10) 0.0224(10) $-0.0013(8) 0.0045(8) 0.0019(8)$ C4B 0.0340(11) 0.0397(12) 0.0280(11) -0.0084(9) 0.0041(9) 0.0055(9) C7A 0.0276(10) 0.0375(11) 0.0238(10) 0.0001(8) 0.0093(8) -0.0015(8) C7B 0.0390(12) 0.0478(14) 0.0415(13) 0.0084(10) 0.0213(10) 0.0000(10)
C10A 0.0257(9) 0.0265(9) 0.0200(9) -0.0003(7) 0.0042(7) 0.0007(8) C10B 0.0357(11) 0.0300(11) 0.0221(10) -0.0027(8) 0.0046(8) -0.0019(9) N1 0.0289(12) 0.0243(13) 0.0218(14) -0.0004(9) 0.0077(11) 0.0037(9) N4 0.0294(17) 0.0269(17) 0.0198(15) 0.0025(11) 0.0017(10) 0.0007(11) N7 0.0211(15) 0.0439(16) 0.0229(16) $-0.0005(12) 0.0051(11) 0.0014(11)$ $\mathrm{N} 10$ 0.0259(13) 0.0256(12) 0.0216(12) $-0.0023(9)$ 0.0043(9) 0.0026(9) C2 0.0353(15) 0.0324(15) 0.0187(13) 0.0052(11) 0.0033(11) 0.0037(13) C3 0.0292(14) 0.0369(15) 0.0217(13) 0.0018(11) 0.0014(11) 0.0053(11) C5 0.0228(13) 0.0480(18) 0.0246(15) -0.0030(13) 0.0030(11) -0.0046(12) C6 0.0211(13) 0.0518(18) 0.0311(15) -0.0001(14) 0.0055(11) 0.0014(12) C8 0.0277(14) 0.0498(18) 0.0297(15) $-0.0068(13) 0.0160(12) 0.0007(13)$ C9 0.0336(15) 0.0374(16) 0.0307(15) -0.0073(12) 0.0091(12) 0.0061(12) C11 0.0420(16) 0.0224(13) 0.0255(14) -0.0004(11) 0.0062(13) 0.0033(11) C12 0.0406(15) 0.0268(14) 0.0282(15) 0.0018(11) 0.0113(13) 0.0075(11) B1 0.023(3) 0.038(4) 0.052(4) 0.006(3) 0.011(3) 0.001(3) F11 0.0256(17) 0.039(2) 0.065(3) 0.0040(18) 0.0108(17) 0.0052(15) F12 0.039(2) 0.0349(19) 0.057(2) -0.0030(15) 0.0024(16) 0.0015(16) F13 0.0269(17) 0.0512(18) 0.080(3) 0.006(2) 0.0230(19) 0.0034(13) F14 0.0480(16) 0.071(2) 0.0383(16) 0.0097(14) -0.0011(13) 0.0095(14) B2 0.037(4) 0.031(3) 0.050(4) 0.002(3) 0.015(3) 0.002(3) F21 0.081(2) 0.122(3) 0.0426(18) 0.0012(19) 0.0247(18) -0.033(2) F22 0.0379(18) $0.065(2) 0.078(3) 0.003(2)-0.004(2) 0.0153(15)$ F23 0.037(2) 0.058(3) 0.052(3) -0.009 (2) 0.0194(17) 0.003(2) F24 0.064(4) 0.036(2) 0.171(6) $-0.009(3) 0.053(4) 0.000(2)$

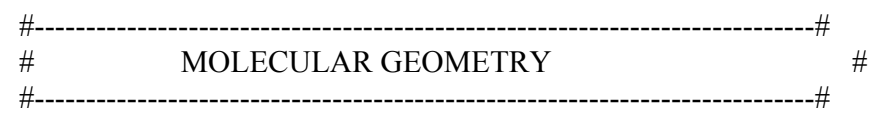

_geom_special_details All esds (except the esd in the dihedral angle between two 1.s. planes) are estimated using the full covariance matrix. The cell esds are taken into account individually in the estimation of esds in distances, angles and torsion angles; correlations between esds in cell parameters are only used when they are defined by crystal symmetry. An approximate (isotropic) treatment of cell esds is used for estimating esds involving 1.s. planes.

loop_

_geom_bond_atom_site_label_1 _geom_bond_atom_site_label_2 geom_bond_distance _geom_bond_site_symmetry_2 geom_bond_publ_flag

$\mathrm{Cu} 1 \mathrm{~N} 1 \mathrm{~A} 2.050(11)$ ? Cu1 N4 2.060(4) . ? Cu1 N7 2.062(3) . ? Cu1 N4A 2.064(15).? Cu1 N10 2.065(2) .?

Cu1 N10A 2.075(10) ? Cu1 N1 2.099(3). ? Cu1 N7A 2.128(12) . ? Cul Cl5 2.3618(5).? C11 C1B 1.791(2).? $\mathrm{Cl} 2 \mathrm{C} 4 \mathrm{~B} 1.792(2)$. ? Cl3 C7B 1.791(2) . ? Cl4 C10B 1.783(2) . ? C1A N1 1.480(3).? C1A C1B 1.525(3) . ? C1A N1A 1.546(11) .?
C1A H1A1 0.99.?

C1A H1A2 0.99.?

C1B H1B1 0.99.?

C1B H1B2 0.99 . ?

C4A N4 1.479(4) . ?

C4A N4A 1.510(15) .?

C4A C4B 1.522(3).?

C4A H4A1 0.99.?

C4A H4A2 0.99.?

C4B H4B1 0.99.?

C4B H4B2 0.99.?

C7A C7B 1.521(3) .?

C7A N7A 1.533(12).?

C7A H7A1 0.99.?

C7A H7A2 0.99.?
C7A N7 1.477(4) . ?
C7B H7B1 0.99.?

C7B H7B2 0.99.?

C10A N10 1.473(3) . ?

C10A C10B 1.522(3).?

C10A N10A 1.567(9). ?

C10A H10A 0.99.?

C10A H10B 0.99.?

C10B H10C 0.99.?

C10B H10D 0.99.?

N1 C12 1.488(3) .?

N1 C2 1.503(3).?

N4 C3 1.496(4) . ?

N4 C5 1.499(4) .?

N7 C6 1.498(4) . ?

N7 C8 1.510(4) . ?

N10 C9 1.487(3) . ?
N10 C11 1.505(4) . ?

C2 C3 1.507(4) . ?

C2 H2A 0.99.?

C2 H2B 0.99.?

C3 H3A 0.99.?

C3 H3B 0.99. ?

C5 C6 1.518(4). ?

C5 H5A 0.99.?

C5 H5B 0.99.?

C6 H6A 0.99.?

C6 H6B 0.99. ?

C8 C9 1.502(4) . ?

C8 H8A 0.99 .?

C8 H8B 0.99.?

C9 H9A 0.99.?

C9 H9B 0.99 . ? 
C11 C12 1.509(4) . ?

C11 H11A 0.99.?

C11 H11B 0.99 . ?

C12 H12A 0.99 .?

C12 H12B 0.99.?

N1A C2A 1.476(12).?

N1A C12A 1.492(13).?

N4A C5A 1.445(15).?

N4A C3A 1.468(15) .?

N7A C8A 1.459(13) .?

N7A C6A 1.490(13) .?
N10A C9A 1.454(12).?

N10A C11A 1.492(12).?

C2A C3A 1.505(12) . ?

C2A H2A1 0.99.?

C2A H2A2 0.99.?

C3A H3A1 0.99.?

C3A H3A2 0.99.?

C5A C6A 1.526(12) . ?

C5A H5A1 0.99.?

C5A H5A2 0.99.?

C6A H6A1 0.99.?
C6A H6A2 0.99.? C8A C9A 1.533(12) . ?

C8A H8A1 0.99.?

C8A H8A2 0.99. ?

C9A H9A1 0.99.?

C9A H9A2 0.99 . ?

C11A C12A 1.521(11).?

C11A H11C 0.99.?

C11A H11D 0.99.?

C12A H12C 0.99.?

C12A H12D 0.99.?
B1 F12 1.383(4) . ? B1 F14 1.387(4) . ? B1 F11 1.387(4) . ? B1 F13 1.393(4) . ? B2 F24 1.377(4) . ? B2 F23 1.378(4) . ? B2 F22 1.383(4) . ? B2 F21 1.385(4) . ?

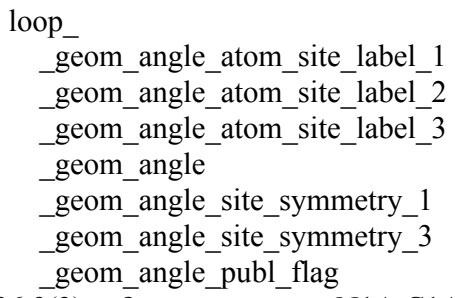

N1A Cu1 N4 96.9(3) .. ?

N1A Cu1 N7 146.0(3) . .?

N4 Cu1 N7 86.51(9) .. ?

N1A Cu1 N4A 86.7(4) .. ?

N4 Cu1 N4A 10.4(3) . . ?

N7 Cu1 N4A 96.0(3)..?

N1A Cu1 N10 72.6(3) . . ?

N4 Cu1 N10 148.95(12) . . ?

N7 Cu1 N10 87.03(9) .. ?

N4A Cu1 N10 145.4(4) . . ?

N1A Cu1 N10A 86.1(3) . . ?

N4 Cu1 N10A 143.9(3) . . ?

N7 Cu1 N10A 72.3(2) .. ?

N4A Cu1 N10A 144.9(5) . .?

N10 Cu1 N10A 14.8(2) . .?

N1A Cu1 N1 12.8(2) . .?

N4 Cu1 N1 85.11(8) . .?

N7 Cu1 N1 149.72(11) . .?

N4A Cu1 N1 74.7(3) . .?

N10 Cu1 N1 85.33(9) . . ?

N10A Cu1 N1 98.5(2) . . ?

N1A Cu1 N7A 146.3(4) . . ?

N4 Cu1 N7A 74.9(3) .. ?

N7 Cu1 N7A 11.7(3) ..?

N4A Cu1 N7A 84.5(4) . . ?

N10 Cu1 N7A 97.4(3) . .?

N10A Cu1 N7A 82.8(4) .. ?

N1 Cu1 N7A 145.4(3) . . ?

N1A Cu1 Cl5 108.4(3) . . ?

N4 Cu1 Cl5 105.91(10) . . ?

N7 Cu1 Cl5 103.03(9) . . ?

N4A Cu1 Cl5 107.7(4) . . ?

N10 Cu1 C15 105.14(7) . . ?

N10A Cu1 Cl5 107.2(3) . .?

N1 Cu1 Cl5 107.25(7) . . ?

N7A Cul Cl5 105.3(3) .. ?

N1 C1A C1B 113.73(18) . .?

N1 C1A N1A 17.5(3) . . ?

C1B C1A N1A 114.5(4) .. ?

N1 C1A H1A1 108.8 . ?

C1B C1A H1A1 108.8 . . ?
N1A C1A H1A1 92.8 . .?

N1 C1A H1A2 108.8 . ? C1B C1A H1A2 108.8 . . ?

N1A C1A H1A2 122.1 . ?

H1A1 C1A H1A2 107.7 . ?

C1A C1B C11 108.44(14) . . ?

C1A C1B H1B1 $110 \ldots$ ?

C11 C1B H1B1 $110 \ldots$ ?

C1A C1B H1B2 $110 \ldots$ ?

C11 C1B H1B2 $110 \ldots$ ?

H1B1 C1B H1B2 108.4 . . ?

N4 C4A N4A 14.3(5) . .?

N4 C4A C4B 114.7(2) .. ?

N4A C4A C4B 113.8(6) . . ?

N4 C4A H4A1 108.6 . .?

N4A C4A H4A1 96.3 . . ?

C4B C4A H4A1 108.6 . . ?

N4 C4A H4A2 108.6 . ?

N4A C4A H4A2 120.6 . .?

C4B C4A H4A2 108.6 . . ?

H4A1 C4A H4A2 107.6 . ?

C4A C4B Cl2 108.11(14) . ?

C4A C4B H4B1 110.1 . . ?

C12 C4B H4B1 110.1 . . ?

C4A C4B H4B2 110.1 . . ?

Cl2 C4B H4B2 110.1 . ?

H4B1 C4B H4B2 108.4 . ?

N7 C7A C7B 114.91(19) . . ?

N7 C7A N7A 16.3(4) ..?

C7B C7A N7A 112.1(5) .. ?

N7 C7A H7A1 108.5 ..?

C7B C7A H7A1 108.5 . . ?

N7A C7A H7A1 95.5 . .?

N7 C7A H7A2 108.5 . ?

C7B C7A H7A2 108.5 . . ?

N7A C7A H7A2 123.1 . . ?

H7A1 C7A H7A2 107.5 . . ?

C7A C7B Cl3 109.59(15) . . ?

C7A C7B H7B1 109.8 . . ?

C13 C7B H7B1 109.8 . . ?

C7A C7B H7B2 109.8 . ?
C13 C7B H7B2 109.8 . . ?

H7B1 C7B H7B2 108.2 . ? N10 C10A C10B 112.45(17) . . ? N10 C10A N10A 19.8(3) ..? C10B C10A N10A 113.5(4) . . ?

N10 C10A H10A 109.1 . . ? C10B C10A H10A 109.1 . . ? N10A C10A H10A 90.9 . . ?

N10 C10A H10B 109.1 . . ? C10B C10A H10B 109.1 . . ? N10A C10A H10B $124 \ldots$ ? H10A C10A H10B 107.8 . . ? C10A C10B Cl4 108.53(13). . ? C10A C10B H10C $110 \ldots$ ? Cl4 C10B H10C $110 \ldots$ ? C10A C10B H10D $110 \ldots$ ? Cl4 C10B H10D $110 \ldots$ ? H10C C10B H10D 108.4 .. ? C1A N1 C12 113.1(2) . . ? C1A N1 C2 112.50(19) . . ? C12 N1 C2 110.0(2) ..? C1A N1 Cu1 111.87(16) . .? C12 N1 Cu1 101.52(16) . ? $\mathrm{C} 2 \mathrm{~N} 1 \mathrm{Cu} 1$ 107.25(18) . . ? C4A N4 C3 114.5(2) . . ? C4A N4 C5 111.3(2) ..? C3 N4 C5 110.0(3) .. ? C4A N4 Cu1 111.4(2) . . ? C3 N4 Cu1 101.7(2) . . ? C5 N4 Cu1 107.5(2)..? C7A N7 C6 113.6(2) . . ? C7A N7 C8 110.6(2) . .? C6 N7 C8 110.2(3)...? C7A N7 Cu1 113.87(19) . . ? C6 N7 Cu1 101.99(18) . . ? C8 N7 Cu1 106.11(19) . . ? C10A N10 C9 112.5(2) . . ? C10A N10 C11 110.63(19) . . ? C9 N10 C11 111.0(2) . . ? C10A N10 Cu1 113.71(15) . . ? C9 N10 Cu1 100.37(16) . . ?
C11 N10 Cu1 108.16(17) . . ? N1 C2 C3 109.8(2) ..? N1 C2 H2A 109.7 . . ? C3 C2 H2A 109.7 . . ? N1 C2 H2B 109.7 . . ? C3 C2 H2B 109.7 . . ? H2A C2 H2B 108.2 . ? N4 C3 C2 109.6(2) . . ? N4 C3 H3A 109.8 . . ? C2 C3 H3A 109.8 . . ? N4 C3 H3B 109.8 . . ? C2 C3 H3B 109.8 . . ? H3A C3 H3B 108.2 . . ? N4 C5 C6 110.7(2) . . ? N4 C5 H5A 109.5 . .? C6 C5 H5A 109.5 . .? N4 C5 H5B 109.5 .. ? C6 C5 H5B 109.5 .. ? H5A C5 H5B 108.1 . . ? N7 C6 C5 109.9(2) ..? N7 C6 H6A 109.7 . . ? C5 C6 H6A 109.7 . . ? N7 C6 H6B 109.7 . . ? C5 C6 H6B 109.7 .. ? H6A C6 H6B 108.2 . . ? C9 C8 N7 110.7(2) . . ? C9 C8 H8A 109.5 . . ? N7 C8 H8A 109.5 . .? C9 C8 H8B 109.5 .. ? N7 C8 H8B 109.5 ..? H8A C8 H8B 108.1 .. ? N10 C9 C8 110.6(2) . . ? N10 C9 H9A 109.5 . . ? C8 C9 H9A 109.5 . . ? N10 C9 H9B 109.5 . . ? C8 C9 H9B 109.5 .. ? H9A C9 H9B 108.1 . . ? N10 C11 C12 110.1(2) . . ? N10 C11 H11A 109.6 . .? C12 C11 H11A 109.6 . ? N10 C11 H11B 109.6 . .? 
C12 C11 H11B 109.6. . ? H11A C11 H11B 108.2 . ? N1 C12 C11 110.1(2) . . ? N1 C12 H12A 109.6 . ? C11 C12 H12A 109.6 . . ? N1 C12 H12B 109.6 . .? C11 C12 H12B 109.6 . ? H12A C12 H12B 108.2 . ? C2A N1A C12A 112.6(9) .. ? C2A N1A C1A 111.2(7) . . ? C12A N1A C1A 111.3(7) . . ? C2A N1A Cu1 101.5(6) . . ? C12A N1A Cu1 108.3(7) . . ? C1A N1A Cu1 111.5(6) . . ? C5A N4A C3A 113.6(11)..? C5A N4A C4A 114.0(9) . . ? C3A N4A C4A 106.7(9) . . ? C5A N4A Cu1 105.3(8) . . ? C3A N4A Cu1 107.2(8) . . ? C4A N4A Cu1 109.8(8) . . ? C8A N7A C6A 112.4(9) . . ? C8A N7A C7A 115.5(8) . . ? C6A N7A C7A 110.3(7) . . ? C8A N7A Cu1 103.3(7) . . ? C6A N7A Cu1 106.8(7) . . ?
C7A N7A Cu1 108.0(6) . . ? C9A N10A C11A 109.7(8) . . ?

C9A N10A C10A 114.2(7) . . ?

C11A N10A C10A 110.5(6). ?

C9A N10A Cu1 110.9(6) . . ? C11A N10A Cu1 101.9(6) . . ?

C10A N10A Cu1 109.0(5) . . ?

N1A C2A C3A 111.5(7) . .?

N1A C2A H2A1 109.3 . . ?

C3A C2A H2A1 109.3 . .?

N1A C2A H2A2 109.3 . . ?

C3A C2A H2A2 109.3 . . ?

H2A1 C2A H2A2 $108 \ldots$ ?

N4A C3A C2A 110.1(9) . . ?

N4A C3A H3A1 109.6 . .?

C2A C3A H3A1 109.6 . . ?

N4A C3A H3A2 109.6 . . ?

C2A C3A H3A2 109.6 . .?

H3A1 C3A H3A2 108.2 . . ?

N4A C5A C6A 110.5(8) . . ?
N4A C5A H5A1 109.5 .. ? C6A C5A H5A1 109.5 . . ? N4A C5A H5A2 109.5 .. ? C6A C5A H5A2 109.5 . . ? H5A1 C5A H5A2 108.1 .. ? N7A C6A C5A 110.3(8) . . ? N7A C6A H6A1 109.6 . . ? C5A C6A H6A1 109.6 . . ? N7A C6A H6A2 109.6 . .? C5A C6A H6A2 109.6 . . ? H6A1 C6A H6A2 108.1 .. ? N7A C8A C9A 109.6(7) . . ? N7A C8A H8A1 109.7 . . ? C9A C8A H8A1 109.7 . . ? N7A C8A H8A2 109.7 . . ? C9A C8A H8A2 109.7 . . ? H8A1 C8A H8A2 108.2 . ? N10A C9A C8A 110.3(7) . . ? N10A C9A H9A1 109.6 . . ? C8A C9A H9A1 109.6 . . ? N10A C9A H9A2 109.6 . . ? C8A C9A H9A2 109.6 . . ? H9A1 C9A H9A2 108.1 . . ? N10A C11A C12A 109.9(7) . . ? N10A C11A H11C 109.7 ..?
C12A C11A H11C 109.7 . . ? N10A C11A H11D 109.7 . . ? C12A C11A H11D 109.7 . . ? H11C C11A H11D 108.2 . .? N1A C12A C11A 109.3(7) ..? N1A C12A H12C 109.8 . ? C11A C12A H12C 109.8 . .? N1A C12A H12D 109.8 . . ? C11A C12A H12D 109.8 . .? H12C C12A H12D 108.3 . . ? F12 B1 F14 109.8(4) . . ? F12 B1 F11 109.9(4) .. ? F14 B1 F11 109.0(4) . . ? F12 B1 F13 109.6(3) . . ? F14 B1 F13 109.5(3) . . ? F11 B1 F13 109.0(4) . . ? F24 B2 F23 110.8(4) . . ? F24 B2 F22 111.3(4) . .? F23 B2 F22 110.4(4) .. ? F24 B2 F21 109.5(4) .. ? F23 B2 F21 106.5(4) . . ? F22 B2 F21 108.2(4) . . ?

\section{CIF data for 3c:}

\#\#CIF_1.1

\# CIF produced by WinGX routine CIF_UPDATE

\# Created on 2003-06-09 at 14:26:27

\# Using CIFtbx version 2.6.2 16 Jun 1998

\# Dictionary name : cif_core.dic

\# Dictionary vers : 2.2

\# Request file : c: \wingx \files $\backslash$ archive.dat

\# CIF files read : 1jf100 dreduc gaussian sortav struct

data_ljf100

_audit_creation_date 2003-06-09T14:26:27-00:00

_audit_creation_method 'WinGX routine CIF_UPDATE'

audit_conform_dict_name cif_core.dic

-audit_conform_dict_version $2 .{ }^{-}$

_audit_conform_dict_location ftp://ftp.iucr.org/pub/cif_core.dic _publ_requested_category

FM

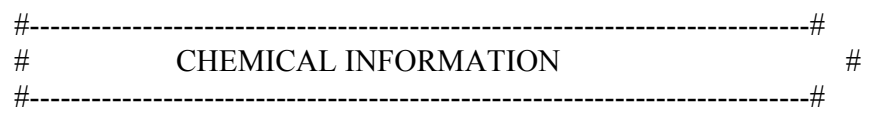

_chemical_name_systematic

;

?

;

_chemical_formula_moiety _chemical_formula_sum
'C12 $\mathrm{H} 25 \mathrm{Cl} 3 \mathrm{Cu}$ N3 O, F6 P' 'C12 H25 Cl3 Cu F6 N3 O P' _chemical_formula_weight

_chemical_compound_source

542.21

'synthesis as described'

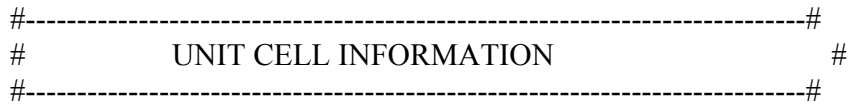

_symmetry_cell_setting

_symmetry_space_group_name_H-M _symmetry_space_group_name_Hall _symmetry_Int_Tables_number

monoclinic 'P 1 21/c 1' '-P 2ybc' 14

loop_

_symmetry_equiv_pos_as_xyz

'x, y, z'

'-x, y+1/2, $-z+1 / 2$ '

'-x, -y, -z'

'x, -y-1/2, z-1/2'

_cell_length_a

_cell_length_b

cell_length_c

_cell_angle_alpha

_cell_angle_beta

-cell_angle_gamma

cell volume

_cell_formula_units_Z

cell_measurement_temperature

_cell_measurement_reflns_used

_cell_measurement_theta_min

_cell_measurement_theta_max

$8.0906(2)$
$18.3838(4)$
$13.7631(3)$
90
$93.5190(10)$
90
$2043.21(8)$
4
$150(2)$
5608
1
30.034

